\title{
Antinutritional factors in plant foods: Potential health benefits and adverse effects
}

\author{
Habtamu Fekadu Gemede ${ }^{1,2,{ }^{*}}$, Negussie Ratta ${ }^{3}$ \\ ${ }^{1}$ Center for Food Science and Nutrition, Addis Ababa University, P.O. Box 1176, Addis Ababa, Ethiopia \\ ${ }^{2}$ Food Technology and Process Engineering Department, Wollega University, P.O.Box: 395, Nekemte, Ethiopia. \\ ${ }^{3}$ Department of Chemistry, Addis Ababa University, P.O.Box: 1176, Addis Ababa Ethiopia
}

\section{Email address:}

fekadu_habtamu@yahoo.com (H. F. Gemede),simbokom@gmail.com (H. F. Gemede)

\section{To cite this article:}

Habtamu Fekadu Gemede, Negussie Ratta. Antinutritional Factors in Plant Foods: Potential Health Benefits and Adverse Effects. International Journal of Nutrition and Food Sciences. Vol. 3, No. 4, 2014, pp. 284-289. doi: 10.11648/j.ijnfs.20140304.18

\begin{abstract}
Anti-nutritional factors are compounds which reduce the nutrient utilization and/or food intake of plants or plant products used as human foods and they play a vital role in determining the use of plants for humans. This paper is aimed to review the updated scientific information regarding the potential health benefits and adverse effects associated with major antinutritional factors found in plant foods. Antinutrients in plant foods are responsible for deleterious effects related to the absorption of nutrients and micronutrients. However, some antinutrients may exert beneficial health effects at low concentrations. For example, phytic acid, lectins, tannins, saponins, amylase inhibitors and protease inhibitors have been shown to reduce the availability of nutrients and cause growth inhibition. However, when used at low levels, phytate, lectins, tannins, amylase inhibitors and saponins have also been shown to reduce the blood glucose and insulin responses to starchy foods and/or the plasma cholesterol and triglycerides. In addition, phytates, tannins, saponins, protease inhibitors, goetrogens and oxalates have been related to reduce cancer risks. This implies that anti-nutrients might not always harmful even though lack of nutritive value. Despite of this, the balance between beneficial and hazardous effects of plant bioactives and anti-nutrients rely on their concentration, chemical structure, time of exposure and interaction with other dietary components. Due to this, they can be considered as anti-nutritional factors with negative effects or non-nutritive compounds with positive effects on health.
\end{abstract}

Keywords: Anti-nutritional factors, Potential Health Benefits, Adverse Health Effects, Plant Foods

\section{Introduction}

Anti-nutritional factors are a chemical compounds synthesized in natural food and / or feedstuffs by the normal metabolism of species and by different mechanisms (for example inactivation of some nutrients, diminution of the digestive process or metabolic utilization of food/feed) which exerts effect contrary to optimum nutrition [1]. Such chemical compounds, are frequently, but not exclusively associated with foods and feeding stuffs of plant origin. These anti-nutritional factors are also known as 'secondary metabolites' in plants and they have been shown to be highly biologically active. These secondary metabolites are secondary compound produced as side products of processes leading to the synthesis of primary metabolites. One major factor limiting the wider food utilization of many tropical plants is the ubiquitous occurrence in them of a diverse range of natural compounds capable of precipitating deleterious effects in man, and animals compound which act to reduce nutrient utilization and/or food intake are often referred to as anti-nutritional factors [2].

Antinutrients are chemicals which have been evolved by plants for their own defense, among other biological functions and reduce the maximum utilization of nutrients especially proteins, vitamins, and minerals, thus preventing optimal exploitation of the nutrients present in a food and decreasing the nutritive value. Some of these plant chemicals have been shown to be deleterious to health or evidently advantageous to human and animal health if consumed at appropriate amounts [3].

There have been several reviews in recent years about the antinutritional factors found in foods. Most of them, however, deal with specific properties or 
beneficial effects for specific antinutritional factors found in a foods and / or feeds. However, this review was aimed to assess updated scientific information of the potential health benefits and adverse effects of major antinutritional factors in plant foods.

\section{Antinutritional Factors in Plant Foods}

\subsection{Tannins}

The word tannin is very old and reflects a traditional technology. Tanning was the word used in the scientific literature to describe the process of transforming raw animal hides or skins into durable, nonputrescible leathers by using plant extracts from different plant parts [10]. Tannin is an astringent, bitter plant polyphenolic compound that either binds or precipitates proteins and various other organic compounds including amino acids and alkaloids [4] The term tannin refers to the use of tannins in tanning animal hides into leather; however, the term is widely applied to any large polyphenolic compound containing sufficient hydroxyls and other suitable groups to form strong complexes with proteins and other macromolecules. Tannins have molecular weights ranging from 500 to over 3000 [5].

Tannins are heat stable and they decreased protein digestibility in animals and humans, probably by either making protein partially unavailable or inhibiting digestive enzymes and increasing fecal nitrogen. Tannins are known to be present in food products and to inhibit the activities of trypsin, chemotrypsin, amylase and lipase, decrease the protein quality of foods and interfere with dietary iron absorption [6].

Tannins are known to be responsible for decreased feed intake, growth rate, feed efficiency and protein digestibility in experimental animals. If tannin concentration in the diet becomes too high, microbial enzyme activities including cellulose and intestinal digestion may be depressed [9]. Tannins also form insoluble complexes with proteins and the tannin-protein complexes may be responsible for the antinutritional effects of tannin containing foods [7, 8].

\subsection{Phytate}

Phytate (is also known as Inositol hexakisphosphate (InsP6)) is the salt form of phytic acid, are found in plants, animals and soil. It is primarily present as a salt of the mono- and divalent cations $\mathrm{K}+, \mathrm{Mg} 2+$, and $\mathrm{Ca} 2+$ and accumulates in the seeds during the ripening period. Phytate is regarded as the primary storage form of both phosphate and inositol in plant seeds and grains. In addition, phytate has been suggested to serve as a store of cations, of high energy phosphoryl groups, and, by chelating free iron, as a potent natural anti-oxidant [10].

Phytate is ubiquitous among plant seeds and grains, comprising 0.5 to 5 percent $(\mathrm{w} / \mathrm{w})$ [22]. The phosphorus bound to phytate is not typically bio-available to any animal that is non-ruminant. Ruminant animals, such as cows and sheep, chew, swallow, and then regurgitate their food. This regurgitated food is known as cud and is chewed a second time. Due to an enzyme located in their first stomach chamber, the rumen, these animals are able to separate, and process the phosphorus in phytates. Humans and other non-ruminant animals are unable to do so [11].

Phytate works in a broad pH-region as a highly negatively charged ion, and therefore its presence in the diet has a negative impact on the bioavailability of divalent, and trivalent mineral ions such as $\mathrm{Zn}^{2+}, \mathrm{Fe}^{2+/ 3+}, \mathrm{Ca}^{2+}, \mathrm{Mg}^{2+}$, $\mathrm{Mn}^{2+}$, and $\mathrm{Cu}^{2+}$. Whether or not high levels of consumption of phytate-containing foods will result in mineral deficiency will depend on what else is being consumed. In areas of the world where cereal proteins are a major and predominant dietary factor, the associated phytate intake is a cause for concern [12].

\subsection{Oxalate}

A salt formed from oxalic acid is known as an Oxalate: for example, Calcium oxalate, which has been found to be widely distributed in plants. Strong bonds are formed between oxalic acid, and various other minerals, such as Calcium, Magnesium, Sodium, and Potassium. This chemical combination results in the formation of oxalate salts. Some oxalate salts, such as sodium and potassium, are soluble, whereas calcium oxalate salts are basically insoluble. The insoluble calcium oxalate has the tendency to precipitate (or solidify) in the Kidneys or in the Urinary tract, thus forming sharp-edged calcium oxalate crystals when the levels are high enough. These crystals play a role to the formation of kidney stones formation in the urinary tract when the acid is excreted in the urine [15].

Oxalate is an anti-nutrient which under normal conditions is confined to separate compartments. However, when it is processed and/or digested, it comes into contact with the nutrients in the gastrointestinal tract [16]. When released, oxalic acid binds with nutrients, rendering them inaccessible to the body. If food with excessive amounts of oxalic acid is consumed regularly, nutritional deficiencies are likely to occur, as well as severe irritation to the lining of the gut. In ruminants oxalic acid is of only minor significance as an anti-nutritive factor since ruminal microflora can readily metabolize soluble oxalates, and to a lesser extent even insoluble $\mathrm{Ca}$ oxalate. While the importance of the anti-nutritive activity of oxalic acid has been recognized for over fifty years it may be a subject of interest to nutritionists in the future $[17,18]$.

Oxalic acid forms water soluble salts with $\mathrm{Na}^{+}, \mathrm{K}+$, and $\mathrm{NH} 4+$ ions, it also binds with $\mathrm{Ca} 2+, \mathrm{Fe} 2+$, and $\mathrm{Mg} 2+$ rendering these minerals unavailable to animals. However $\mathrm{Zn} 2+$ appears to be relatively unaffected. In plants with a cell sap of approximately $\mathrm{pH} 2$, such as some species of Oxalis and rumex oxalate exists as the acid oxalate (HC2O4), primarily as acid potassium oxalate. In plants with a cell sap of approximately $\mathrm{pH} 6$, such as some plants 
of goosefoot family it exists as oxalate (C2O4)2- ion usually as soluble sodium oxalate and insoluble calcium and magnesium oxalates. Calcium oxalate is insoluble at a neutral or alkaline $\mathrm{pH}$, but freely dissolves in acid [19].

\subsection{Saponins}

Saponins are secondary compounds that are generally known as non-volatile, surface active compounds which are widely distributed in nature, occurring primarily in the plant kingdom. The name 'saponin' is derived from the Latin word sapo which means 'soap', because saponin molecules form soap-like foams when shaken with water. They are structurally diverse molecules that are chemically referred to as triterpene and steroid glycosides. They consist of nonpolar aglycones coupled with one or more monosaccharide moieties. This combination of polar and non-polar structural elements in their molecules explains their soap-like behaviour in aqueous solutions.

The structural complexity of saponins results in a number of physical, chemical, and biological properties, which include sweetness and bitterness, foaming and emulsifying properties, pharmacological and medicinal properties, haemolytic properties, as well as antimicrobial, insecticidal, and molluscicidal activities. Saponins have found wide applications in beverages and confectionery, as well as in cosmetics and pharmaceutical products. Due to the presence of a lipid-soluble aglycone and water soluble sugar chain(s) in their structure (amphiphilic nature), saponins are surface active compounds with detergent, wetting, emulsifying, and foaming properties [2].

Saponins were treated as toxic because they seemed to be extremely toxic to fish and cold-blooded animals and many of them possessed strong hemolytic activity. Saponins, in high concentrations, impart a bitter taste and astringency in dietary plants. The bitter taste of saponin is the major factor that limits its use. In the past, saponins were recognized as antinutrient constituents, due to their adverse effects such as for growth impairment and reduce their food intake due to the bitterness and throat-irritating activity of saponins. In addition, saponins were found to reduce the bioavailability of nutrients and decrease enzyme activity and it affects protein digestibility by inhibit various digestive enzymes such as trypsin and chymotrypsin [21].

Saponins are attracting considerable interest as a result of their beneficial effects in humans. Recent evidence suggests that saponins possess hypocholesterolemic, immunostimulatory, and anticarcinogenic properties. In addition, they reduce the risk of heart diseases in humans consuming a diet rich in food legumes containing saponins. Saponin-rich foods are important in human diets to control plasma cholesterol, preventing peptic ulcer, osteoporosis and to reduce the risk of heart disease. Saponins are used as adjuvants in viral (e.g., Quillaja saponaria-21) and bacterial vaccine (e.g., Quillaja saponins) applications . A high saponin diet can be used in the inhibition of dental caries and platelet aggregation, in the treatment of hypercalciuria in humans, and as an antidote against acute lead poisoning.
In epidemiological studies, saponins have been shown to have an inverse relationship with the incidence of renal stones [22].

\subsection{Lectins}

Lectin comes from the Latin word "legere", which means "to select". Lectins have the ability to bind carbohydrates. Nowadays, proteins that can agglutinate red blood cells with known sugar specificity are referred to as "lectins" (Fereidoon S., 2014). The name "hemagglutinins" is used when the sugar specificity is unknown. Lectins and hemagglutinins are proteins/glycoproteins, which have at least one non-catalytic domain that exhibits reversible binding to specific monosaccharides or oligosaccharides. They can bind to the carbohydrate moieties on the surface of erythrocytes and agglutinate the erythrocytes, without altering the properties of the carbohydrates (Sze Kwan Lam \& Tzi Bun Ng, 2013).

Lectins are glycoproteins widely distributed in legumes and some certain oil seeds (including soybean) which possess an affinity for specific sugar molecules and are characterized by their ability to combine with carbohydrate membrane receptors. Lectins have the capability to directly bind to the intestinal muscosa, interacting with the enterocytes and interfering with the absorption and transportation of $0.01 \%$ free gossypol within some lowgossypol cotton nutrients (particularly carbohydrates) during digestion and causing epithelial lesions within the intestine. Although lectins are usually reported as being labile, their stability varies between plant species, many lectins being resistant to inactivation by dry heat and requiring the presence of moisture for more complete destruction (Boehm and Huck, 2009).

Lectins have become the focus of intense interest for biologists and in particular for the research and applications in agriculture and medicine. These proteins with unique characteristics have found use in diverse fields of biology and as more lectins are being isolated and their role in nature elucidated, they continue to occupy an important place in agricultural and therapeutic areas of research (Sze Kwan Lam \& Tzi Bun Ng, 2013).

Lectins are carbohydrate binding proteins present in most plants, especially seeds like cereals, beans, etc., in tubers like potatoes and also in animals. Lectins selectively bind carbohydrates and importantly, the carbohydrate moieties of the glycoproteins that decorate the surface of most animal cells. Dietary lectins act as protein antigens which bind to surface glycoproteins (or glycolipids) on erythrocytes or lymphocytes (Sauvion et al., 2004). They function as both allergens and hemagglutinins and are present in small amounts in $30 \%$ of foods, more so in a whole-grain diet. Lectins have potent in vivo effects. When consumed in excess by sensitive individuals, they can cause 3 primary physiological reactions: they can cause severe intestinal damage disrupting digestion and causing nutrient deficiencies; they can provoke $\operatorname{IgG}$ and IgM antibodies causing food allergies and other immune responses (Boehm 
and Huck, 2009) and they can bind to erythrocytes, simultaneously with immune factors, causing hemagglutination and anemia. Of the 119 known dietary lectins, about half are panhemagglutinins, clumping all blood types. The remainder are blood-type specific. In general, lectins alter host resistance to infection, cause failure to thrive and can even lead to death in experimental animals (Vasconcelos and Oliveira, 2004).

Lectin comes from the Latin word "legere", which means "to select". Lectins have the ability to bind carbohydrates. Nowadays, proteins that can agglutinate red blood cells with known sugar specificity are referred to as "lectins" (Fereidoon S., 2014). The name "hemagglutinins" is used when the sugar specificity is unknown. Lectins and hemagglutinins are proteins/glycoproteins, which have at least one non-catalytic domain that exhibits reversible binding to specific monosaccharides or oligosaccharides. They can bind to the carbohydrate moieties on the surface of erythrocytes and agglutinate the erythrocytes, without altering the properties of the carbohydrates [23].

Lectins have become the focus of intense interest for biologists and in particular for the research and applications in agriculture and medicine. These proteins with unique characteristics have found use in diverse fields of biology and as more lectins are being isolated and their role in nature elucidated, they continue to occupy an important place in agricultural and therapeutic areas of research [25].

Lectins are carbohydrate binding proteins present in most plants, especially seeds like cereals, beans, etc., in tubers like potatoes and also in animals. Lectins selectively bind carbohydrates and importantly, the carbohydrate moieties of the glycoproteins that decorate the surface of most animal cells. Dietary lectins act as protein antigens which bind to surface glycoproteins (or glycolipids) on erythrocytes or lymphocytes [26]. They function as both allergens and hemagglutinins and are present in small amounts in $30 \%$ of foods, more so in a whole-grain diet. Lectins have potent in vivo effects. When consumed in excess by sensitive individuals, they can cause 3 primary physiological reactions: they can cause severe intestinal damage disrupting digestion and causing nutrient deficiencies; they can provoke $\operatorname{IgG}$ and IgM antibodies causing food allergies and other immune responses [27] and they can bind to erythrocytes, simultaneously with immune factors, causing hemagglutination and anemia. Of the 119 known dietary lectins, about half are panhemagglutinins, clumping all blood types. The remainder are blood-type specific. In general, lectins alter host resistance to infection, cause failure to thrive and can even lead to death in experimental animals [28].

\subsection{Alkaloids}

Alkaloids are one of the largest groups of chemical compounds synthesised by plants and generally found as salts of plant acids such as oxalic, malic, tartaric or citric acid. Alkaloids are small organic molecules, common to about 15 to 20 per cent of all vascular plants, usually comprising several carbon rings with side chains, one or more of the carbon atoms being replaced by a nitrogen. They are synthesized by plants from amino acids. Decarboxylation of amino acids produces amines which react with amine oxides to form aldehydes. The characteristic heterocyclic ring in alkaloids is formed from Mannich-type condensation from aldehyde and amine groups [27].

The chemical type of their nitrogen ring offers the means by which alkaloids are subclassified: for example, glycoalkaloids (the aglycone portion) glycosylated with a carbohydrate moiety. They are formed as metabolic byproducts. Insects and hervibores are usually repulsed by the potential toxicity and bitter taste of alkaloids [28, 29].

Alkaloids are considered to be anti-nutrients because of their action on the nervous system, disrupting or inappropriately augmenting electrochemical transmission. For instance, consumption of high tropane alkaloids will cause rapid heartbeat, paralysis and in fatal case, lead to death. Uptake of high dose of tryptamine alkaloids will lead to staggering gate and death. Indeed, the physiological effects of alkaloids have on humans are very evident. Cholinesterase is greatly inhibited by glycoalkaloids, which also cause symptoms of neurological disorder. Other toxic action includes disruption of the cell membrane in the gastrointestinal tract [30].

\subsection{Protease Inhibitors}

Protease inhibitors are widely distributed within the plant kingdom, including the seeds of most cultivated legumes and cereals. Protease inhibitors are the most commonly encountered class of antinutritional factors of plant origin. Protease inhibitors have the ability to inhibit the activity of proteolytic enzymes within the gastrointestinal tract of animals. Due to their particular protein nature, protease inhibitors may be easily denatured by heat processing although some residual activity may still remain in the commercially produced products. The antinutrient activity of protease inhibitors is associated with growth inhibition and pancreatic hypertrophy. Potential beneficial effects of protease inhibitors remain unclear, although lower incidences of pancreatic cancer have been observed in populations where the intake of soybean and its products is high [33]. While protease inhibitors have been linked with pancreatic cancer in animal studies, they may also act as anticarcinogenic agents. The Bowman-Birk inhibitors derived from soybean have been shown to inhibit or prevent the development of chemically-induced cancer of the liver, lung, colon, oral and esophagus [31].

Trypsin inhibitor and chymotrypsin inhibitor are protease inhibitors occurring in raw legume seeds. Trypsin inhibitors that inhibit the activity of the enzymes trypsin and chymotrypsin in the gut, thus preventing protein digestion, are found in many plant species mainly in different grain legumes. Trypsin inhibitors are a unique class of proteins found in raw soybeans that inhibit protease enzymes in the digestive tract by forming indigestible complexes with 
dietary protein. These complexes are indigestible even in the presence of high amounts of digestive enzymes. Protease inhibitors reduce trypsin activity and to a lesser extent chymotrypsin; therefore impairing protein digestion by monogastric animals and some young ruminant animals [32].

\subsection{Cyanogenic Glycosides}

The cyanogenic glycosides belong to the products of secondary metabolism, to the natural products of plants. These compounds are composed of an a-hydroxynitrile type aglycone and of a sugar moiety (mostly D-glucose). Cyanogenic glucosides (a-hydroxynitrile glucosides) are derived from the five protein amino acids Val, Ile, Leu, Phe and Tyr and from the nonproteinogenic amino acid cyclopentenyl glycine. Although derived from six different building blocks, they constitute a very small class with around 50 different known structures. A number of plant species produce hydrogen cyanide $(\mathrm{HCN})$ from cyanogenic glycosides when they are consumed. These cyanogens are glycosides of a sugar, often glucose, which is combined with a cyanide containing aglycone. Cyanogenic glucosides are classified as phytoanticipins. Their general function in plants is dependent on activation by b-glucosidases to release toxic volatile $\mathrm{HCN}$ as well as a ketones or aldehydes to fend off herbivore and pathogen attack [34].

\section{Conclusion}

Antinutritional factors in foods are responsible for the deleterious effects that are related to the absorption of nutrients and micronutrients which may interfere with the function of certain organs. Most of these antinutritional factors are present in foods of plant origin. Thus, the presence of cyanogenic glycosides, protease inhibitors, lectins, tannins, alkaloids, and saponnins in foods may induce undesirable effects in humans if their consumption exceeds an upper limit. Certain harmful effects might also be due to the breakdown products of these compounds. However, some antinutritional factors as well as their breakdown products may possess beneficial health effects if present in small amounts. The mechanism through which the antinutritional and beneficial effects of food antinutritional factors are exerted is the same. Health risk factors associated with antinutritional factors include: lack of knowledge of the tolerance levels to these compounds in the human organism, little available information on the degree of variation of individual risks and little knowledge with respect to the influence of environmental factors on the detoxification capacity of the human organism.

\section{Acknowledgement}

Authors would like to acknowledge all the Authors of the articles, we used as a references in preparing this review paper. The authors have no conflict of interest.

\section{References}

[1] Soetan K. and Oyewol O. (2009). The need for adequate processing to reduce the antinutritional factors in plants used as human foods and animal feeds: A review. African Journal of Food Science Vol. 3 (9), pp. 223-232.

[2] Shanthakumari, S., Mohan, V. and Britto, J. (2008). Nutritional evaluation and elimination of toxic principles in wild yam (Dioscorea spp.). Tropical and Subtropical Agroecosystems, (8), 319 - 225.

[3] Ugwu, F. M. and Oranye, N. A. (2006). Effects of some processing methods on the toxic components ofAfrican breadfruit (Treculia qfricana). African Journal 0/Biotechnology 5,2329-2333.

[4] Redden, R.J., Chen, W., \& Sharma, B. (2005). Chickpea Breeding and Management. United Kingdom: CABI.

[5] Muzquiz, M., Burbano, C., Cuadrado, C., and Martin, M. (2000). Analytical methods for determination of compounds with no nutritive value. In Handbook on Common Bean Related Laboratory Methods (p. 11-26). Spain:Galicia.

[6] Felix, J.P., and Mello, D. (2000). Farm Animal Metabolism and Nutrition. United Kingdom: CABI.

[7] Panhwar F. (2005). Anti-nutritional factors in oil seeds as aflatoxin in ground nut. Retrieved from www.ChemLin.com.

[8] Kyriazakis I. and Whittenmore C.T. (2006). Whittenmore's Science and Practice of Pig Production. Oxford: WileyBlackwell.

[9] Aletor VA. (2005). Anti-nutritional factors as nature's paradox in food and nutrition securities. Inaugural lecture series 15, delivered at The Federal University of Technology, Akure (FUTA).

[10] Mueller I. (2001). Analysis of hydrolysable tannins. Anim Feed Sci Technol 91:3-20.

[11] Harold (2004). On food and cooking: the science and lore of the kitchen. New York: Scribner. pp 714. ISBN 0-68480001-2.

[12] Mueller I. (2001). Analysis of hydrolysable tannins. Anim Feed Sci Technol 91:3-20.

[13] Mushtaq M. (2000). Nutritional evaluation of soybean meal varying in urease and trypsin inhibitor activity. Thesis disertation, North Carolina State University, Raleigh, NC.

[14] Muzquiz, M., Burbano, C., Cuadrado, C., and Martin, M. (2000). Analytical methods for determination of compounds with no nutritive value. In Handbook on Common Bean Related Laboratory Methods (p. 11-26). Spain:Galicia.

[15] Nachbar M.S., Oppenheim J.D. and Thomas J.O. (2000). Lectins in the US diet: Isolation and characterization of a lectin from the tomato (Lycopersicon). J. Biol. Chem., 255:2056.

[16] Noonan, S. C. and Savage, G. P. (1999). Oxalic acid and its effects on humans. Asia pacific Journal of Clinical Nutrition, $8,64-74$. 
[17] Oladimeji M. O., Akindahunsi A. A., and Okafor, A. F. (2000). Investigation of the bioavailability of zinc and calcium from some tropical tubers. Nahrung, 44, 136-137 $(\mathrm{Nr} 2, \mathrm{~S})$.

[18] Liebman M., Al-Wahsh I.A. (2011). Probiotics and other key determinants of dietary oxalate absorption. Adv. Nutr. 2, 254-260.

[19] Liener I. E. (2005). Implications of antinutritional components in soybean foods. Food Sci. 34:31.

[20] Liener I.E. and Kakade M.L. (1980). Protease inhibitors. In: Toxic constituents of plant food stuffs (Editor: I.E. Liener) Academic Press, New York, pp: 7-71.

[21] Liener, I.E., (2003). Phytohemagglutinins: Their nutritional significance. J. Agric. Food Chem., 22: 17.

[22] Loewus FA.(2002). Biosynthesis of phytate in food grains and seeds. In: Reddy NR, Sathe SK (Eds.). Food Phytates. CRC Press, Boca Raton Florida, pp 53-61.

[23] Luo J., Litherland A.J., Sahlu T., Puchala R., Lachica M. and Goetsch, A.L. (2000). Effects of mimosine on fiber shedding, follicle activity and fiber regrowth in Spanish goats. J. Anim. Sci., 78: 1551-1555.

[24] kernels. Grasas of Aceite (Espana), 44: 235-242.

[25] Dost K., \& Tokul O. (2006). Determination of phytic acid in wheat and wheat products by reversed phase high performance liquid chromatography, Analytica Chimica Acta, 558, 26-27.

[26] FAO (1995). Sorghum and Millets in Human Nutrition.
Food and Agriculture Organization of the United Nations, Italy, Rome.

[27] Felix, J.P., and Mello, D. (2000). Farm Animal Metabolism and Nutrition. United Kingdom: CABI.

[28] Fereidoon S. (2014). Beneficial Health Effects and Drawbacks of Antinutrients and Phytochemicals in Foods. Appl Microbiol Biotechnol 97:45-55.

[29] Fereidoon S., (2012). Antinutrients and Phytochemicals in Food. Developed from a symposium sponsored by the Division of Agricultural and Food Chemistry at the 210th National Meeting of the American Chemical Society, Chicago, Illinoi. ACS symposium series, ISSN 0097-6156;2

[30] Fernando R. Pinto MDP. and Pathmeswaran A. (2012). Goitrogenic Food and Prevalence of Goitre in Sri Lanka. J. Food Sci., 41: 1076-1081.

[31] Finotti E., Bertone A and Vivanti V. (2006). Balance between nutrients and anti-nutrients in nine Italian potato cultivars. Food Chemistry 99, 698-701.

[32] Friedman M, Henika P.R. and Mackey B.E. (2003). Effect of feeding solanidine, solasodine and tamatidine to nonpregnant and pregnant mice. Food and Chemical Toxicology $41,61-71$

[33] Giri A.P. and Kachole, M.S. (2004). Amylase inhibitors of pigeon pea (Cajanus cajan) seeds. Phytochemistry, 47: 197202.

[34] Golden M. (2009). Nutrient requirements of moderately malnourished populations of children. Food Nutr Bull. 